\title{
Existence of Positive Periodic Solutions for a Nonautonomous Generalized Predator-Prey System with Time Delay in Two-Patch Environment
}

\author{
Huilan Wang, ${ }^{1}$ Zhengqiu Zhang, ${ }^{2}$ and Weiping Zhou ${ }^{1}$ \\ ${ }^{1}$ Department of Mathematics and Physics, Nanhua University, Hengyang 421001, China \\ ${ }^{2}$ Department of Applied Mathematics, Hunan University, Changsha 410082, China
}

Correspondence should be addressed to Huilan Wang, huilan07@163.com

Received 25 November 2011; Accepted 26 January 2012

Academic Editor: Vimal Singh

Copyright (C) 2012 Huilan Wang et al. This is an open access article distributed under the Creative Commons Attribution License, which permits unrestricted use, distribution, and reproduction in any medium, provided the original work is properly cited.

By using continuation theorem of coincidence degree theory, sufficient conditions of the existence of positive periodic solutions are obtained for a generalized predator-prey system with diffusion and delays. In this paper, we construct a V-function to make the prior estimation for periodic solutions, which makes the discussion more concise. Moreover, to compute the mapping's topological degree, a polynomial function matrix is constructed straightforwardly as a homotopic mapping for the generalized one, which improves the methods of computation on topological degree for a generalized mapping.

\section{Introduction}

In the study of dynamic population models, which are represented by differential equations, to elaborate the realistic factors into models, sometimes one needs to consider the effects caused by various of factors such as delays, diffusion, and others. The global characteristics (including persistent, stable or attractive, oscillatory, and chaotic behavior) of such differential system have been an intensive subject in biomathematics (see e.g., [1-6]). One important aspect is the existence of positive periodic solutions ([7-17]), which imply the system is periodic.

In the literature available, the methods of studying the existence of periodic solutions of a periodic system often fall into one of the following three categories: (1) combining the results of persistence of system with Horn fixed point theorem (usually for a system with delay) or Brower fixed point theorem (usually for a system without delay), the existence 
results of periodic solutions are obtained $([7,8])$; $(2)$ employing other kinds of fixed point theorems (usually for one dimension system), the differential model is investigated by transforming it into equivalent integral one ([9-11]); (3) by virtue of the theory of topological degree, especially by using Mawhin's Continuation theorem of coincidence degree (usually for high dimension system), much significant work has been done ([12-17]). However, for a generalized high-dimension system, no matter by fixed point theorems or by Mawhin's continuation theorem of coincidence degree, it is very difficult to accomplish the work. The relevant literature is seldom to find. Zhang considered a generalized prey-predator system with delay ([16]), where the generalized mapping's degree is computed step by step and a new technique of computation on topological degree is presented.

It is well known that the key and the difficult points are the prior estimation for solutions, and the topological degree's computation of generalized mapping as Mawhin's continuation theorem is applied. In this paper, we investigate a generalized model of one predator competing for two preys, which takes the nonautonomous form:

$$
\begin{aligned}
& \dot{x}_{1}(t)=x_{1}(t) f_{1}\left(t, x_{1}(t), x_{3}(t)\right)+D_{1}(t)\left(x_{2}(t)-x_{1}(t)\right), \\
& \dot{x}_{2}(t)=x_{2}(t) f_{2}\left(t, x_{2}(t), x_{3}(t)\right)+D_{2}(t)\left(x_{1}(t)-x_{2}(t)\right), \\
& \dot{x}_{3}(t)=x_{3}(t)\left[-g\left(t, x_{3}(t)\right)+c_{1}(t) p_{1}\left(x_{1}\left(t-\tau_{1}\right)\right)+c_{2}(t) p_{2}\left(x_{2}\left(t-\tau_{2}\right)\right)\right],
\end{aligned}
$$

where $x_{i}$ denotes the density of the prey in the $i$ th patch; $x_{3}$ represents the total predator population for both patches; $\tau_{i}>0$ is a constant and $D_{i}(t)$ is a positive continuous function and denotes the dispersal rate; $p_{i}$ is the functional response of the predator population on the prey in the $i$ th patch, and $c_{i}$ is the conversion ratio of prey into predator; $D_{i}(t), c_{i}(t), f_{i}\left(t, x_{i}, x_{3}\right)$ and $g\left(t, x_{3}\right)$ are continuous functions in $t \in[0,+\infty)$ with a common period $\omega>0 ; f_{i}\left(t, x_{i}, x_{3}\right)$ and $g\left(t, x_{3}\right)$ are differentiable with the other variables; $i=1,2$. The model is transformed from an autonomous one (see [6]). In [6], sufficient conditions for the permanence and the existence of positive attractive equilibrium are derived.

Our purpose is to obtain sufficient conditions for the existence of positive periodic solutions associated with system (1.1) and to consider whether delays and diffusion have effect on the results. What is more important is to present some new techniques in prior estimation of solutions and computation on topological degree. In this paper, we construct a V-function to make the prior estimation, which makes the proof more concise. Furthermore, we select a polynomial function matrix as a homotopic mapping to the generalized one, which plays a key role in the computation of topological degree.

In a biological sense, we take the initial conditions:

$$
x_{i}(s)=\varphi_{i}(s), \quad s \in[-\tau, 0], \quad \varphi_{i}(0)>0, \quad i=1,2,3,
$$

where $\tau=\max \left\{\tau_{1}, \tau_{2}\right\}$.

In system (1.1), we assume that

$\left(\mathrm{H}_{1}\right)$ for $t \geq 0, \partial f_{i}\left(t, x_{i}, x_{3}\right) / \partial x_{i}<0, \partial f_{i}\left(t, x_{i}, x_{3}\right) / \partial x_{3}<0, i=1,2$, and $\left(\partial g\left(t, x_{3}\right)\right) / \partial x_{3}>$ 0 ;

$\left(\mathrm{H}_{2}\right) p_{i}(x)$ is continuous and $p_{i}(0)=0, p_{i}^{\prime}(x)>0, i=1,2$. 
For a positive continuous $\omega$-periodic function $f(t)$, we define

$$
f^{u}=\max _{t \in[0, \omega]} f(t), \quad f^{l}=\min _{t \in[0, \omega]} f(t), \quad \bar{f}=\frac{1}{\omega} \int_{0}^{\omega} f(t) d t .
$$

In the following sections, we derived the sufficient conditions for the existence of positive periodic solutions and show that the delays and diffusion have no effect on the result.

\section{Main Result and Proof}

We first introduce some notion of the continuation theorem of coincidence degree theory and the lemma formulated in [18].

Let $X, Z$ be Banach spaces, let $L:$ Dom $L \subset X \rightarrow Z$ be a linear mapping, and let $N: X \rightarrow Z$ be a continuous mapping. The mapping $L$ will be called a Fredholm mapping of index zero if dimker $L=\operatorname{codimim} L<+\infty$ and $\operatorname{Im} L$ is closed in $Z$. Let $P: X \rightarrow X$ and $Q: Z \rightarrow Z$ be two projectors such that $\operatorname{Im} P=\operatorname{Ker} L$ and $\operatorname{Im} L=\operatorname{Ker} Q=\operatorname{Im}(I-Q)$. It follows that $L /(\operatorname{Dom} L \cap \operatorname{Ker} P):(I-P) X \rightarrow I m L$ is invertible. We denote the inverse of that map by $K_{P}$. For an open bounded subset $\Omega$ of X, the mapping $N$ will be called $L$-compact on $\bar{\Omega}$ if $Q N(\bar{\Omega})$ is bounded and $K_{P}(I-P) N: \bar{\Omega} \rightarrow X$ is compact. Since $\operatorname{Im} Q$ is isomorphic to Ker $L$, there exists an isomorphism $J: \operatorname{Im} Q \rightarrow \operatorname{Ker} L$.

Lemma 2.1. Let L be a Fredholm mapping of index zero and $N$ be L-compact on $\bar{\Omega}$. Suppose

(a) for each $\lambda \in(0,1)$ and $x \in \partial \Omega, L x \neq \lambda N x$;

(b) for each $x \in \operatorname{Ker} L \bigcap \partial \Omega, Q N x \neq 0$;

(c) Brower degree $\operatorname{deg}_{B}(J Q N, \Omega \cap \operatorname{Ker} L, 0) \neq 0$.

Then $L x=N x$ has at least one solution in $\operatorname{Dom} L \cap \bar{\Omega}$.

Theorem 2.2. Assume that there exist four positive constants $M, N, m, n$ such that

$$
\begin{aligned}
& \text { (1) for } t \in R, f_{i}(t, M, 0) \leq 0 \text { and }-g(t, N)+c_{1}(t) p_{1}(M)+c_{2}(t) p_{2}(M)<0, i=1,2 \text {; } \\
& \text { (2) for } t \in R, f_{i}(t, m, N) \geq 0 \text { and }-g(t, n)+c_{1}(t) p_{1}(m)+c_{2}(t) p_{2}(m)>0, i=1,2 \text {. }
\end{aligned}
$$

Then system (1.1) has at least one positive w-periodic solution.

Proof. Denote $x_{i}(t)=\exp \left[u_{i}(t)\right](i=1,2,3)$; then system (1.1) can be rewritten as

$$
\begin{aligned}
& \dot{u}_{1}(t)=f_{1}\left(t, e^{u_{1}(t)}, e^{u_{3}(t)}\right)+D_{1}(t)\left(e^{u_{2}(t)-u_{1}(t)}-1\right), \\
& \dot{u}_{2}(t)=f_{2}\left(t, e^{u_{2}(t)}, e^{u_{3}(t)}\right)+D_{2}(t)\left(e^{u_{1}(t)-u_{2}(t)}-1\right), \\
& \dot{u}_{3}(t)=-g\left(t, e^{u_{3}(t)}\right)+c_{1}(t) p_{1}\left(e^{u_{1}\left(t-\tau_{1}\right)}\right)+c_{2}(t) p_{2}\left(e^{u_{2}\left(t-\tau_{2}\right)}\right) .
\end{aligned}
$$


Clearly, if system (2.1) has an $\omega$-periodic solution $\left(u_{1}^{*}(t), u_{2}^{*}(t), u_{3}^{*}(t)\right)^{T}$, then system (1.1) has a positive $\omega$-periodic solution $\left(\exp \left[u_{1}^{*}(t)\right], \exp \left[u_{2}^{*}(t)\right], \exp \left[u_{3}^{*}(t)\right]\right)^{T}$. We define

$$
\begin{gathered}
X=Z=\left\{u(t)=\left(u_{1}(t), u_{2}(t), u_{3}(t)\right)^{T} \in C\left(R, R^{3}\right), u(t+\omega)=u(t)\right\}, \\
\|u\|=\left\|\left(u_{1}(t), u_{2}(t), u_{3}(t)\right)^{T}\right\|=\sum_{i=1}^{3} \max _{t \in[0, \omega]}\left|u_{i}(t)\right|, \quad u \in X(\text { or } Z) .
\end{gathered}
$$

Then $X$ and $Z$ are Banach spaces with the norm $\|\cdot\|$.

Let

$$
\begin{gathered}
N(u, \lambda)=\left[\begin{array}{c}
f_{1}\left(t, e^{u_{1}(t)}, e^{u_{3}(t)}\right)+\lambda D_{1}(t)\left(e^{u_{2}(t)-u_{1}(t)}-1\right) \\
f_{2}\left(t, e^{u_{2}(t)}, e^{u_{3}(t)}\right)+\lambda D_{2}(t)\left(e^{u_{1}(t)-u_{2}(t)}-1\right) \\
-g\left(t, e^{u_{3}(t)}\right)+c_{1}(t) p_{1}\left(e^{u_{1}\left(t-\tau_{1}\right)}\right)+c_{2}(t) p_{2}\left(e^{u_{2}\left(t-\tau_{2}\right)}\right)
\end{array}\right], \quad u \in X, \lambda \in(0,1), \\
L u=u^{\prime}=\frac{d u(t)}{d t}, \\
P u=\frac{1}{\omega} \int_{0}^{\omega} u(t) \mathrm{d} t, \quad u \in X, \quad Q z=\frac{1}{\omega} \int_{0}^{\omega} z(t) d t, \quad z \in Z .
\end{gathered}
$$

Then, $L$ is a Fredholm mapping of index zero and the generalized inverse of $L$ is $K_{P}: \operatorname{Im} L \rightarrow$ Ker $P \cap$ Dom $L$, which is given by

$$
K_{p}(z)=\int_{0}^{t} z(s) d s-\frac{1}{\omega} \int_{0}^{\omega} \int_{0}^{t} z(s) d s d t
$$

Hence

$$
Q N(u, \lambda)=\left[\begin{array}{l}
\frac{1}{\omega} \int_{0}^{\omega} F_{1}(s) d s \\
\frac{1}{\omega} \int_{0}^{\omega} F_{2}(s) d s \\
\frac{1}{\omega} \int_{0}^{\omega} F_{3}(s) d s
\end{array}\right]
$$

where

$$
\begin{gathered}
F_{1}(s)=f_{1}\left(s, e^{u_{1}(s)}, e^{u_{3}(s)}\right)+\lambda D_{1}(s)\left(e^{u_{2}(s)-u_{1}(s)}-1\right), \\
F_{2}(s)=f_{2}\left(s, e^{u_{2}(s)}, e^{u_{3}(s)}\right)+\lambda D_{2}(s)\left(e^{u_{1}(s)-u_{2}(s)}-1\right), \\
F_{3}(s)=-g\left(s, e^{u_{3}(s)}\right)+c_{1}(s) p_{1}\left(e^{u_{1}\left(s-\tau_{1}\right)}\right)+c_{2}(s) p_{2}\left(e^{u_{2}\left(s-\tau_{2}\right)}\right) .
\end{gathered}
$$


Consequently,

$$
K_{p}(I-Q) N(u, \lambda)=\left[\begin{array}{l}
\int_{0}^{t} F_{1}(s) d s-\frac{1}{\omega} \int_{0}^{\omega} \int_{0}^{t} F_{1}(s) d s d t+\left(\frac{1}{2}-\frac{t}{\omega}\right) \int_{0}^{\omega} F_{1}(s) d s \\
\int_{0}^{t} F_{2}(s) d s-\frac{1}{\omega} \int_{0}^{\omega} \int_{0}^{t} F_{2}(s) d s d t+\left(\frac{1}{2}-\frac{t}{\omega}\right) \int_{0}^{\omega} F_{2}(s) d s \\
\int_{0}^{t} F_{3}(s) d s-\frac{1}{\omega} \int_{0}^{\omega} \int_{0}^{t} F_{3}(s) d s d t+\left(\frac{1}{2}-\frac{t}{\omega}\right) \int_{0}^{\omega} F_{3}(s) d s
\end{array}\right] .
$$

By Arzela-Ascoli theorem, we can verify that the map $K_{P}(I-Q) N(\bar{\Omega})$ is compact. Thus, $N$ is $L$ - compact on $\bar{\Omega}$ for any open bounded $\Omega \subset X$. Since $\operatorname{Im} Q=\operatorname{Ker} L$, the isomorphism $J$ is the identical mapping.Corresponding to the operator equation $L x=\lambda N(x, \lambda), \lambda \in(0,1)$, we have

$$
\begin{aligned}
& \dot{u}_{1}(t)=\lambda\left[f_{1}\left(t, e^{u_{1}(t)}, e^{u_{3}(t)}\right)+\lambda D_{1}(t)\left(e^{u_{2}(t)-u_{1}(t)}-1\right)\right], \\
& \dot{u}_{2}(t)=\lambda\left[f_{2}\left(t, e^{u_{2}(t)}, e^{u_{3}(t)}\right)+\lambda D_{2}(t)\left(e^{u_{1}(t)-u_{2}(t)}-1\right)\right], \\
& \dot{u}_{3}(t)=\lambda\left[-g\left(t, e^{u_{3}(t)}\right)+c_{1}(t) p_{1}\left(e^{u_{1}\left(t-\tau_{1}\right)}\right)+c_{2}(t) p_{2}\left(e^{u_{2}\left(t-\tau_{2}\right)}\right)\right] .
\end{aligned}
$$

Assume that $u=u(t) \in X$ is a solution of (2.8) for a certain $\lambda \in(0,1)$. Then there exist $\xi_{i}, \eta_{i} \in[0, \omega](i=1,2,3)$ such that

$$
\begin{aligned}
u_{i}\left(\xi_{i}\right)=\max _{t \in[0, \omega]} u_{i}(t), & u_{i}\left(\eta_{i}\right)=\min _{t \in[0, \omega]} u_{i}(t), \\
u_{i}^{\prime}\left(\xi_{i}\right)=0, & u_{i}^{\prime}\left(\eta_{i}\right)=0 .
\end{aligned}
$$

Therefore, we obtain the equations

$$
\begin{gathered}
f_{1}\left(\xi_{1}, e^{u_{1}\left(\xi_{1}\right)}, e^{u_{3}\left(\xi_{1}\right)}\right)+\lambda D_{1}\left(\xi_{1}\right)\left(e^{u_{2}\left(\xi_{1}\right)-u_{1}\left(\xi_{1}\right)}-1\right)=0, \\
f_{2}\left(\xi_{2}, e^{u_{2}\left(\xi_{2}\right)}, e^{u_{3}\left(\xi_{2}\right)}\right)+\lambda D_{2}\left(\xi_{2}\right)\left(e^{u_{1}\left(\xi_{2}\right)-u_{2}\left(\xi_{2}\right)}-1\right)=0, \\
-g\left(\xi_{3}, e^{u_{3}\left(\xi_{3}\right)}\right)+c_{1}\left(\xi_{3}\right) p_{1}\left(e^{u_{1}\left(\xi_{3}-\tau_{1}\right)}\right)+c_{2}\left(\xi_{3}\right) p_{2}\left(e^{u_{2}\left(\xi_{3}-\tau_{2}\right)}\right)=0
\end{gathered}
$$

and equations

$$
\begin{gathered}
f_{1}\left(\eta_{1}, e^{u_{1}\left(\eta_{1}\right)}, e^{u_{3}\left(\eta_{1}\right)}\right)+\lambda D_{1}\left(\eta_{1}\right)\left(e^{u_{2}\left(\eta_{1}\right)-u_{1}\left(\eta_{1}\right)}-1\right)=0, \\
f_{2}\left(\eta_{2}, e^{u_{2}\left(\eta_{2}\right)}, e^{u_{3}\left(\eta_{2}\right)}\right)+\lambda D_{2}\left(\eta_{2}\right)\left(e^{u_{1}\left(\eta_{2}\right)-u_{2}\left(\eta_{2}\right)}-1\right)=0 \\
-g\left(\eta_{3}, e^{u_{3}\left(\eta_{3}\right)}\right)+c_{1}\left(\eta_{3}\right) p_{1}\left(e^{u_{1}\left(\eta_{3}-\tau_{1}\right)}\right)+c_{2}\left(\eta_{3}\right) p_{2}\left(e^{u_{2}\left(\eta_{3}-\tau_{2}\right)}\right)=0 .
\end{gathered}
$$


To make estimation of $u_{i}\left(\xi_{i}\right)(i=1,2,3)$, we define a V-function as $\bar{V}(t)=\max \left\{u_{1}(t), u_{2}(t)\right\}$. Obviously, $\bar{V}\left(\xi_{i}\right)=u_{i}\left(\xi_{i}\right)$ and $\dot{\bar{V}}\left(\xi_{i}\right)=0, \quad i=1,2$. According to the hypothesis and equations (2.10), we have

$$
\begin{aligned}
f_{i}\left(\xi_{i}, e^{\bar{V}\left(\xi_{i}\right)}, 0\right) & >f_{i}\left(\xi_{i}, e^{\bar{V}\left(\xi_{i}\right)}, e^{u_{3}\left(\xi_{i}\right)}\right) \\
& =f_{i}\left(\xi_{i}, e^{u_{i}\left(\xi_{i}\right)}, e^{u_{3}\left(\xi_{i}\right)}\right) \\
& \geq 0 \\
& \geq f_{i}\left(\xi_{i}, M, 0\right), \quad i=1,2
\end{aligned}
$$

which implies that $e^{\bar{V}\left(\xi_{i}\right)}<M$, that is,

$$
u_{i}\left(\xi_{i}\right)<\ln M, \quad i=1,2
$$

The third equation of (2.10) gives

$$
\begin{aligned}
g\left(\xi_{3}, e^{u_{3}\left(\xi_{3}\right)}\right) & =c_{1}\left(\xi_{3}\right) p_{1}\left(e^{u_{1}\left(\xi_{3}-\tau_{1}\right)}\right)+c_{2}\left(\xi_{3}\right) p_{2}\left(e^{u_{2}\left(\xi_{3}-\tau_{2}\right)}\right) \\
& \leq c_{1}\left(\xi_{3}\right) p_{1}\left(e^{u_{1}\left(\xi_{1}\right)}\right)+c_{2}\left(\xi_{3}\right) p_{2}\left(e^{u_{2}\left(\xi_{2}\right)}\right) \\
& <c_{1}\left(\xi_{3}\right) p_{1}(M)+c_{2}\left(\xi_{3}\right) p_{2}(M) \\
& <g\left(\xi_{3}, N\right) .
\end{aligned}
$$

Thus

$$
e^{u_{3}\left(\xi_{3}\right)}<N
$$

or

$$
u_{3}\left(\xi_{3}\right)<\ln N
$$

Similarly, we define $\underline{V}(t)=\min \left\{u_{1}(t), u_{2}(t)\right\}$. Then $\underline{V}\left(\eta_{i}\right)=u_{i}\left(\eta_{i}\right)$, and $\underline{V}\left(\eta_{i}\right)=0, i=1,2$. From (2.11), we have

$$
\begin{aligned}
f_{i}\left(\eta_{i}, e^{\underline{V}\left(\eta_{i}\right)}, N\right) & =f_{i}\left(\eta_{i}, e^{u_{i}\left(\eta_{i}\right)}, N\right) \\
& <f_{i}\left(\eta_{i}, e^{u_{i}\left(\eta_{i}\right)}, e^{u_{3}\left(\eta_{i}\right)}\right) \\
& \leq 0 \\
& <f_{i}\left(\eta_{i}, m, N\right),
\end{aligned}
$$


which implies that

$$
e^{u_{i}\left(\eta_{i}\right)}>m,
$$

that is,

$$
u_{i}\left(\eta_{i}\right)>\ln m
$$

From (2.19), it follows that

$$
\begin{aligned}
g\left(\eta_{3}, e^{u_{3}\left(\eta_{3}\right)}\right)= & c_{1}\left(\eta_{3}\right) p_{1}\left(e^{u_{1}\left(\eta_{3}-\tau_{1}\right)}\right)+c_{2}\left(\eta_{3}\right) p_{2}\left(e^{u_{2}\left(\eta_{3}-\tau_{2}\right)}\right) \\
& \geq c_{1}\left(\eta_{3}\right) p_{1}\left(e^{u_{1}\left(\eta_{1}\right)}\right)+c_{2}\left(\eta_{3}\right) p_{2}\left(e^{u_{2}\left(\eta_{2}\right)}\right) \\
& \geq c_{1}\left(\eta_{3}\right) p_{1}(m)+c_{2}\left(\xi_{3}\right) p_{2}(m) \\
& >g\left(\eta_{3}, n\right) .
\end{aligned}
$$

Hence

$$
e^{u_{3}\left(\eta_{3}\right)}>n,
$$

that is,

$$
u_{3}\left(\eta_{3}\right)>\ln n
$$

Denote

$$
R_{1}=\max \{|\ln m|,|\ln M|\}, \quad R_{2}=\max \{|\ln n|,|\ln N|\} .
$$

In view of (2.13)-(2.22), we have

$$
\left|u_{1}(t)\right|<R_{1}, \quad\left|u_{2}(t)\right|<R_{1}, \quad\left|u_{3}(t)\right|<R_{2}, \quad \forall t \in R .
$$

Clearly, $R_{i}(i=1,2)$ are independent of $\lambda$. Denote $\widetilde{M}=2 R_{1}+R_{2}+R_{0}$, where $R_{0}$ is taken sufficiently large such that the solution $\left(\alpha^{*}, \beta^{*}, \gamma^{*}\right)^{T}$ of the following system:

$$
\begin{gathered}
\frac{1}{\omega} \int_{0}^{\omega} f_{1}\left(s, e^{\alpha}, e^{\gamma}\right) d s=0 \\
\frac{1}{\omega} \int_{0}^{\omega} f_{2}\left(s, e^{\beta}, e^{\gamma}\right) d s=0 \\
\frac{1}{\omega} \int_{0}^{\omega}\left[-g\left(s, e^{\gamma}\right)+c_{1}(s) p_{1}\left(e^{\alpha}\right)+c_{2}(s) p_{2}\left(e^{\beta}\right)\right] d s=0
\end{gathered}
$$


satisfies $\left\|\left(\alpha^{*}, \beta^{*}, \gamma^{*}\right)^{T}\right\|<\widetilde{M}$, provided that system (2.25) has one or a number of solutions. Let $\Omega=\left\{u=\left(u_{1}(x), u_{2}(x), u_{3}(x)\right)^{T} \in X ;\|u\|<\widetilde{M}\right\}$. It is easy to see that the condition (a) of Lemma 2.1 is satisfied.

When $u \in \partial \Omega \cap \operatorname{Ker} L=\partial \Omega \cap R^{3}, u$ is a constant vector in $R^{3}$ with $\sum_{i=1}^{3}\left|u_{i}\right|=\widetilde{M}$. If system (2.25) has one or a number of solutions, then

$$
Q N(u, 0)=\left[\begin{array}{c}
\frac{1}{\omega} \int_{0}^{\omega} f_{1}\left(s, e^{u_{1}}, e^{u_{3}}\right) d s \\
\frac{1}{\omega} \int_{0}^{\omega} f_{2}\left(s, e^{u_{2}}, e^{u_{3}}\right) d s e \\
\frac{1}{\omega} \int_{0}^{\omega}\left[-g\left(s, e^{u_{3}}\right)+c_{1}(s) p_{1}\left(e^{u_{1}}\right)+c_{2}(s) p_{2}\left(e^{u_{2}}\right)\right] d s
\end{array}\right] \neq(0,0,0)^{T} .
$$

If system (2.25) has no solution, then naturally $Q N(u, 0) \neq(0,0,0)^{T}$. Hence, the condition (b) of Lemma 2.1 is satisfied.

Finally, we will prove that condition (c) of Lemma 2.1 is satisfied. To this end, we define a mapping $\psi:$ Dom $L \times[0,1] \rightarrow X$ by

$$
\psi(u, v)=v\left[\begin{array}{c}
\frac{1}{\omega} \int_{0}^{\omega} f_{1}\left(s, e^{u_{1}}, e^{u_{3}}\right) d s \\
\frac{1}{\omega} \int_{0}^{\omega} f_{2}\left(s, e^{u_{2}}, e^{u_{3}}\right) d s \\
\frac{1}{\omega} \int_{0}^{\omega}\left[-g\left(s, e^{u_{3}}\right)+c_{1}(s) p_{1}\left(e^{u_{1}}\right)+c_{2}(s) p_{2}\left(e^{u_{2}}\right)\right] d s
\end{array}\right]+(1-v)\left[\begin{array}{l}
a-e^{u_{1}} \\
a-e^{u_{2}} \\
b-e^{u_{3}}
\end{array}\right],
$$

where $v \in[0,1]$ is a parameter and $a, b$ are two chosen numbers as follows:

$$
m<a<M, \quad n<b<N \text {. }
$$

In the following, we will show that when $u \in \partial \Omega \cap \operatorname{Ker} L, \psi(u, v) \neq(0,0,0)^{T}$.

We consider three possible cases: (1) either of $e^{u_{i}}$ satisfies $e^{u_{i}} \geq M(i=1,2)$, (2) either of $e^{u_{i}}$ satisfies $e^{u_{i}} \leq m(i=1,2)$, and (3) both of $e^{u_{i}}$ satisfy $m<e^{u_{i}}<M(i=1,2)$.

Case 1. Either of $e^{u_{i}}(i=1,2)$ satisfies $e^{u_{i}} \geq M$ : since there exists a constant $t_{1}$ such that

$$
\frac{1}{\omega} \int_{0}^{\omega} f_{i}\left(s, e^{u_{i}}, e^{u_{3}}\right) d s=f_{i}\left(t_{1}, e^{u_{i}}, e^{u_{3}}\right),
$$


then

$$
\begin{aligned}
v f_{i}\left(t_{1}, e^{u_{i}}, e^{u_{3}}\right)+(1-v)\left(a-e^{u_{i}}-b e^{u_{3}}\right. & <v f_{i}\left(t_{1}, e^{u_{i}}, 0\right)+(1-v)\left(a-e^{u_{i}}\right) \\
\leq & v f_{i}\left(t_{1}, M, 0\right)+(1-v)(a-M)<0 .
\end{aligned}
$$

Case 2. Either of $e^{u_{i}}(i=1,2)$ satisfies $e^{u_{i}} \leq m$ : we consider two subcases as follows.

Subcase 1. $e^{u_{3}}<N$. There exists a constant $t_{2}$ and $u_{i}(i=1$ or 2$)$ such that

$$
\frac{1}{\omega} \int_{0}^{\omega} f_{i}\left(s, e^{u_{i}}, e^{u_{3}}\right) d s=f_{i}\left(t_{2}, e^{u_{i}}, e^{u_{3}}\right)
$$

then

$$
\begin{aligned}
v f_{i}\left(t_{2}, e^{u_{i}}, e^{u_{3}}\right)+(1-v)\left(a-e^{u_{i}}\right)>v f_{i}\left(t_{2}, m, N\right) \\
+(1-v)(a-m) \geq v f_{i}\left(t_{2}, m, N\right)+(1-v)(a-m)>0
\end{aligned}
$$

Subcase 2. $e^{u_{3}} \geq N$. Because the other $u_{j}\left(j=2\right.$ or 1 ) must satisfy either $e^{u_{j}} \leq M$ or $e^{u_{j}} \geq M$. The later one is discussed in Case 1 . Then we only consider the subcase when $e^{u_{i}} \leq m$ (i= 1 or 2$)$ and $e^{u_{j}} \leq M(j=2$ or 1$)$. Under these conditions, there exists a constant $t_{3}$ such that

$$
\begin{aligned}
& v\left[-g\left(t_{3}, e^{u_{3}}\right)+c_{1}\left(t_{5}\right) p_{1}\left(e^{u_{1}}\right)+c_{2}\left(t_{5}\right) p_{2}\left(e^{u_{2}}\right)\right]+(1-v)\left(b-e^{u_{3}}\right) \\
& \quad<\left[-g\left(t_{3}, N\right)+c_{1}\left(t_{5}\right) p_{1}(M)+c_{2}\left(t_{5}\right) p_{2}(M)\right]+(1-v)(b-N)<0 .
\end{aligned}
$$

Case 3. Both of $e^{u_{i}}(i=1,2)$ satisfy $m<e^{u_{i}}<M$ : we also consider the following two subcases. Subcase 3. $e^{u_{3}} \geq N$. There exists a constant $t_{4}$ such that

$$
\begin{gathered}
\frac{1}{\omega} \int_{0}^{\omega}\left[-g\left(s, e^{u_{3}}\right)+c_{1}(s) p_{1}\left(e^{u_{1}(s)}\right)+c_{2}(s) p_{2}\left(e^{u_{2}(s)}\right)\right] d s \\
=-g\left(t_{4}, e^{u_{3}}\right)+c_{1}\left(t_{4}\right) p_{1}\left(e^{u_{1}}\right)+c_{2}\left(t_{4}\right) p_{2}\left(e^{u_{2}}\right) .
\end{gathered}
$$

Hence

$$
\begin{aligned}
v & {\left[-g\left(t_{4}, e^{u_{3}}\right)+c_{1}\left(t_{4}\right) p_{1}\left(e^{u_{1}}\right)+c_{2}\left(t_{4}\right) p_{2}\left(e^{u_{2}}\right)\right]+(1-v)\left(b-e^{u_{3}}\right) } \\
& <v\left[-g\left(t_{4}, N\right)+c_{1}\left(t_{4}\right) p_{1}(M)+c_{2}\left(t_{4}\right) p_{2}(M)\right]+(1-v)(b-N) \\
& <0 .
\end{aligned}
$$


Subcase 4. $e^{u_{3}}<N$. If $n<e^{u_{3}}<N$, then $\sum_{i=1}^{3}\left|u_{i}\right|<\widetilde{M}$, which is a contradiction to $\sum_{i=1}^{3}\left|u_{i}\right|=$ $\widetilde{M}$. Hence, $e^{u_{3}} \leq n$. When $m<e^{u_{i}}<M$ and $e^{u_{3}} \leq n$, there exists a $t_{5}$ such that

$$
\begin{aligned}
v & {\left[-g\left(t_{5}, e^{u_{3}}\right)+c_{1}\left(t_{3}\right) p_{1}\left(e^{u_{1}}\right)+c_{2}\left(t_{5}\right) p_{2}\left(e^{u_{2}}\right)\right]+(1-v)\left(b-e^{u_{3}}\right) } \\
& >v\left[-g\left(t_{5}, n\right)+c_{1}\left(t_{5}\right) p_{1}(m)+c_{2}\left(t_{5}\right) p_{2}(m)\right]+(1-v)(b-n) \\
& >0 .
\end{aligned}
$$

To sum up, $\psi(u, v) \neq(0,0,0)^{T}$ when $u \in \partial \Omega \cap \operatorname{Ker} L$.

Since the algebraic equations

$$
\begin{aligned}
& a-x=0, \\
& a-y=0, \\
& b-z=0
\end{aligned}
$$

have a unique solution $\left(x^{*}, y^{*}, z^{*}\right)$ such that

$$
x^{*}=y^{*}=a>0, \quad z^{*}=b>0,
$$

then

$$
\begin{aligned}
& \operatorname{deg}\left(\operatorname{JQN}(u, 0), \Omega \cap \operatorname{Ker} L,(0,0,0)^{T}\right) \\
& \quad=\operatorname{sign}\left|\begin{array}{ccc}
-x^{*} & 0 & 0 \\
0 & -y^{*} & 0 \\
0 & 0 & -z^{*}
\end{array}\right|=\operatorname{sign}\left[-x^{*} y^{*} z^{*}\right]=-1 \neq 0 .
\end{aligned}
$$

This completes the proof of Theorem 2.2.

Remark 2.3. Theorem 2.2 implies that the delays and the diffusion have no effect on the result provided $\left(\mathrm{H}_{1}\right)-\left(\mathrm{H}_{2}\right)$ holds.

\section{Application}

Example 3.1. Consider the system:

$$
\begin{gathered}
\dot{x}_{1}(t)=x_{1}(t)\left[r_{1}(t)-a_{1}(t) x_{1}(t)-b_{1}(t) x_{3}(t)\right]+D_{1}(t)\left(x_{2}(t)-x_{1}(t)\right), \\
\dot{x}_{2}(t)=x_{2}(t)\left[r_{2}(t)-a_{2}(t) x_{2}(t)-b_{2}(t) x_{3}(t)\right]+D_{2}(t)\left(x_{1}(t)-x_{2}(t)\right), \\
\dot{x}_{3}(t)=x_{3}(t)\left[-r_{3}(t)-a_{3}(t) x_{3}+c_{1}(t) x_{1}\left(t-\tau_{1}\right)+c_{2}(t) x_{2}\left(t-\tau_{2}\right)\right],
\end{gathered}
$$


where $\tau_{i}(i=1,2)$ are positive constants and all the coefficients are positive continuous functions with period $\omega$.

It is easy to see that

$$
f_{i}\left(t, x_{i}, x_{3}\right)=r_{i}(t)-a_{i}(t) x_{i}-b_{i}(t) x_{3}, \quad i=1,2 ; \quad g\left(t, x_{3}\right)=r_{3}(t)+a_{3}(t) x_{3} ; \quad p_{i}(x)=x .
$$

Obviously, the assumptions $\left(\mathrm{H}_{1}\right)-\left(\mathrm{H}_{2}\right)$ are satisfied. Let

$$
M=\max \left\{\frac{r_{1}^{u}}{a_{1}^{l}}, \frac{r_{2}^{u}}{a_{2}^{l}}\right\} .
$$

Then

$$
f_{i}(t, M, 0) \leq 0
$$

Let

$$
N=\frac{\left(c_{1}^{u}+c_{2}^{u}\right) M-r_{3}^{l}}{a_{3}^{l}}
$$

then $-g(t, N)+c_{1}(t) M+c_{2}(t) M<0$. While

$$
m=\min \left\{\frac{r_{1}^{l}-b_{1}^{u} N}{a_{1}^{u}}, \frac{r_{2}^{l}-b_{2}^{u} N}{a_{2}^{u}}\right\} .
$$

$f_{i}(t, m, N) \geq 0$. Let

$$
n=\frac{\left(c_{1}^{l}+c_{2}^{l}\right) m-r_{3}^{u}}{a_{3}^{u}}
$$

then $-g(t, n)+c_{1}(t) m+c_{2}(t) m>0$. Therefore, the conditions of Theorem 2.2 are satisfied. We can draw the conclusion that system (3.1) has at least one positive periodic solution.

\section{References}

[1] D. Mukherjee and A. B. Roy, "Uniform persistence and global attractivity theorem for generalized prey-predator system with time delay," Nonlinear Analysis, Theory, Methods and Applications, vol. 38, no. 1, pp. 59-74, 1999.

[2] M. Fan and X. Zou, "Global asymptotic stability of a class of nonautonomous integro-differential systems and applications," Nonlinear Analysis, Theory, Methods and Applications, vol. 57, no. 1, pp. 111$135,2004$.

[3] D. Mukherjee, D. Mitra, S. Ray, and A. B. Roy, "The effect of diffusion on two predators exploiting a resource," BioSystems, vol. 31, no. 1, pp. 49-58, 1993. 
[4] H. I. Freedman and Y. Takeuchi, "Global stability and predator dynamics in a model of prey dispersal in a patchy environment," Nonlinear Analysis, vol. 13, no. 8, pp. 993-1002, 1989.

[5] Y. Kuang, "Predator-prey dynamics in models of prey dispersal in two-patch environments," Mathematical Biosciences, vol. 120, no. 1, pp. 77-98, 1994.

[6] W. Wendi and M. Zhien, "Asymptotic behavior of a predator-prey system with diffusion and delays," Journal of Mathematical Analysis and Applications, vol. 206, no. 1, pp. 191-204, 1997.

[7] S. L. Sun and C. D. Yuan, "Analysis for three-species mixture model with diffusion and ratiodependence," Journal of Systems Science and Mathematical Sciences, vol. 1, no. 25, pp. 87-95, 2005 (Chinese).

[8] Z. D. Teng and L. S. Chen, "The positive periodic solutions of periodic Kolmogonov type systems with delays," Acta Mathematical Application Sinica, vol. 22, pp. 446-456, 1999 (Chinese).

[9] Y. Li, "Positive periodic solutions of first and second order ordinary differential equations," Chinese Annals of Mathematics. Series B, vol. 25, no. 3, pp. 413-420, 2004.

[10] H. Wang, "Positive periodic solutions of functional differential equations," Journal of Differential Equations, vol. 202, no. 2, pp. 354-366, 2004.

[11] Y. Li, P. Liu, and L. Zhu, "Positive periodic solutions of a class of functional differential systems with feedback controls," Nonlinear Analysis, Theory, Methods and Applications, vol. 57, no. 5-6, pp. 655-666, 2004.

[12] Y. Li and Y. Kuang, "Periodic solutions in periodic state-dependent delay equations and population models," Proceedings of the American Mathematical Society, vol. 130, no. 5, pp. 1345-1353, 2002.

[13] Y. H. Fan, W. T. Li, and L. L. Wang, "Periodic solutions of delayed ratio-dependent predatorprey models with monotonic or nonmonotonic functional responses," Nonlinear Analysis: Real World Applications, vol. 5, no. 2, pp. 247-263, 2004.

[14] Z. Q. Zhang and Z. C. Wang, "The existence of a positive periodic solution for a generalized preypredator system with delay," Mathematics Proceedings of Cambridge Philosophy Society, vol. 137, pp. 475-186, 2004.

[15] Z. Yang, "Positive periodic solutions for a class of nonlinear delay equations," Nonlinear Analysis, Theory, Methods and Applications, vol. 59, no. 7, pp. 1013-1031, 2004.

[16] Z. Zhang and H. Wang, "Existence and global attractivity of positive periodic solutions for a generalized predator-prey system with time delay," Mathematical and Computer Modelling, vol. 44, no. 1-2, pp. 188-203, 2006.

[17] H. Fang and Z. Wang, "Existence and global attractivity of positive periodic solutions for delay LotkaVolterra competition patch systems with stocking," Journal of Mathematical Analysis and Applications, vol. 293, no. 1, pp. 190-209, 2004.

[18] R. E. Gains and J. L. Mawhin, Coincidence Degree and Nonlinear Differential Equations, Springer, Berlin, Germany, 1977. 


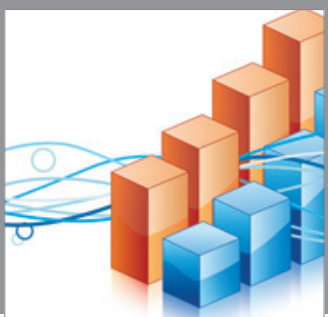

Advances in

Operations Research

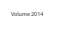

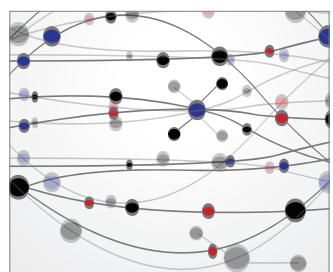

\section{The Scientific} World Journal
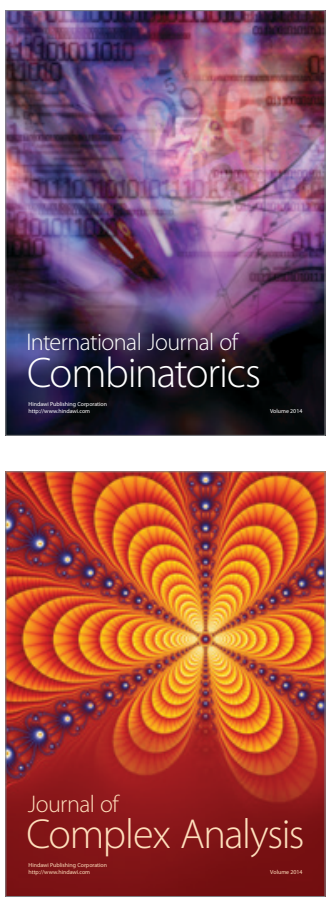

International Journal of

Mathematics and

Mathematical

Sciences
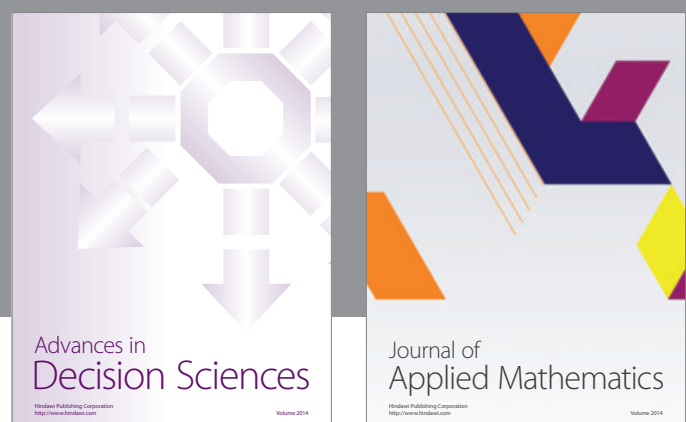

Journal of

Applied Mathematics
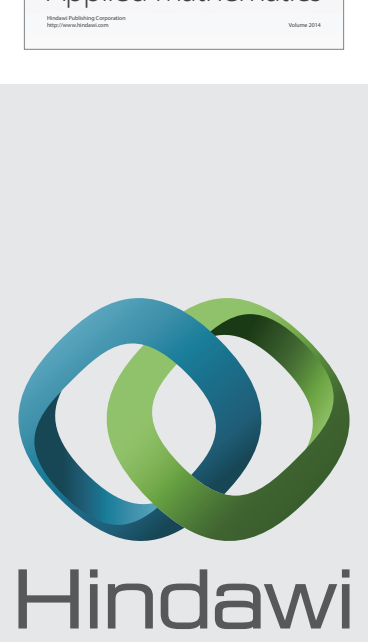

Submit your manuscripts at http://www.hindawi.com
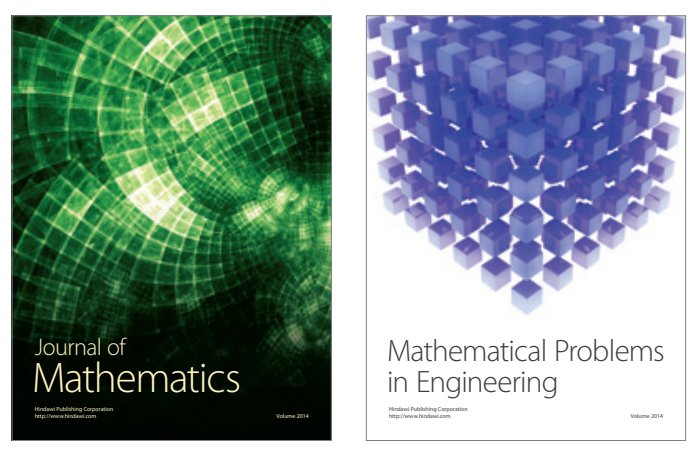

Mathematical Problems in Engineering
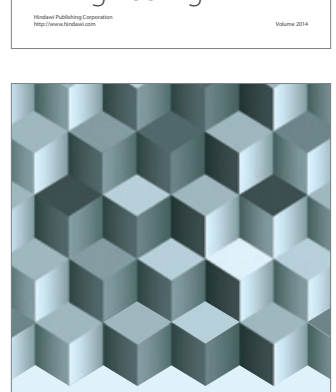

Journal of

Function Spaces
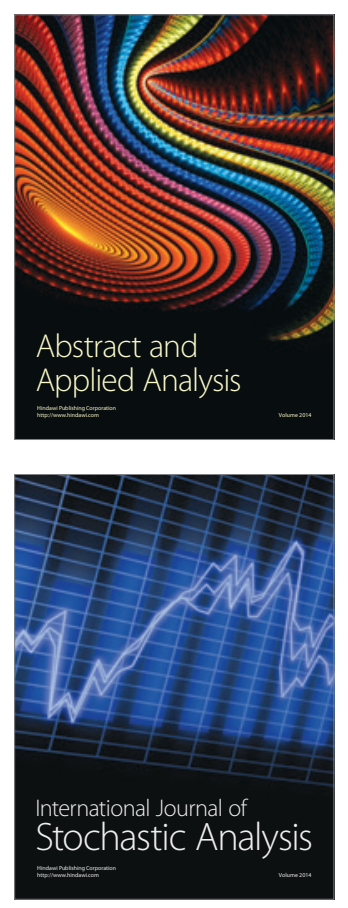

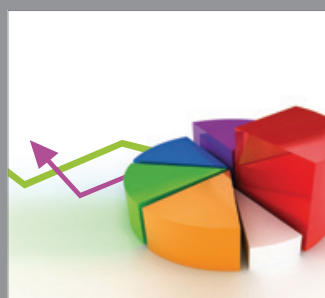

ournal of

Probability and Statistics

Promensencen
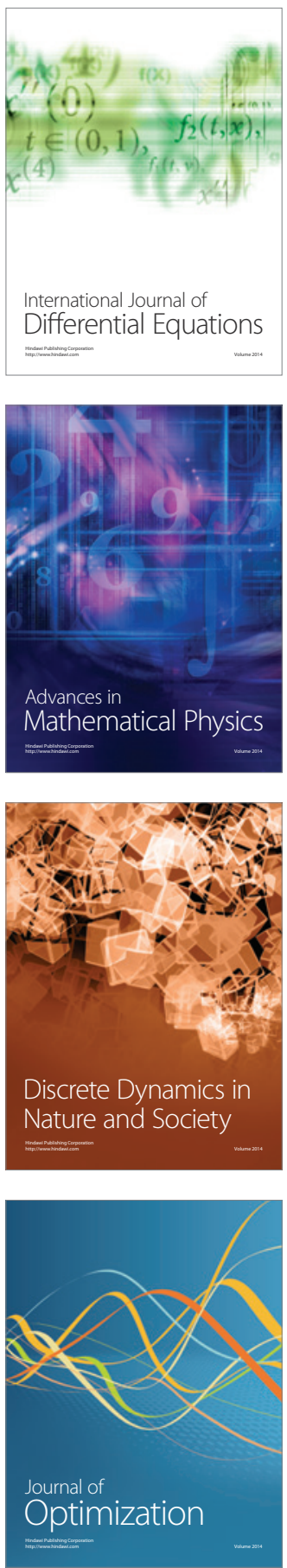